\title{
NEW CONTINUITY ESTIMATES OF GEOMETRIC SUMS
}

\author{
EVGUENI GORDIENKO and JUAN RUIZ DE CHÁVEZ \\ Universidad Autónoma Metropolitana, Unidad Iztapalapa \\ Av. Michaocán y la Purísima s $\mathrm{n}$, Col. Vicentina \\ Apartado Postal 55-534, México 09340, D.F.
}

(Received August, 2000; Revised March, 2002)

\begin{abstract}
The paper deals with sums of a random number of independent and identically distributed random variables. More specifically, we compare two such sums, which differ from each other in the distributions of their summands. New upper bounds (inequalities) for the uniform distance between distributions of sums are established. The right-hand sides of these inequalities are expressed in terms of Zolotarev's and the uniform distances between the distributions of summands. Such a feature makes it possible to consider these inequalities as continuity estimates and to apply them to the study of the stability (continuity) of various applied stochastic models involving geometric sums and their generalizations.
\end{abstract}

Key words: Comparison of Sums of Independent Random Variables, Upper Bounds of Proximity, Uniform Metric, Zolotarev's Metric.

AMS subject classifications: 60E15, 60G50, 60K10.

\section{Introduction}

In this paper we treat random variables defined as follows:

$$
Z=\sum_{k=1}^{\nu} X_{k}
$$

where $X_{1}, X_{2}, \ldots$ are independent, identically distributed random variables (i.i.d. random variables, for short) and $\nu$ is a random variable independent of $\left(X_{k}, k \geq 1\right)$, taking natural values $k=1,2, \ldots$.

Let $p_{k}=P(\nu=k), k=1,2, \ldots$ and $F$ denote the distribution function of $X_{1}$. The problem under consideration is a quantitative comparison of the distribution of $Z$ in (1.1) with the distribution of the sum:

$$
\widetilde{Z}=\sum_{k=1}^{\nu} \widetilde{X}_{k}
$$

where the random variable $\nu$ is the same as in (1.1) and $\widetilde{X}_{1}, \widetilde{X}_{2}, \ldots$, are i.i.d. random variables with a common distribution function $\widetilde{F}$.

In what follows we use the standard definition of the uniform probability metric:

$$
\rho(\xi, \eta)=\rho\left(F_{\xi}, F_{\eta}\right):=\sup _{x \in \mathbb{R}}\left|F_{\xi}(x)-F_{\eta}(x)\right|,
$$


( $F_{\xi}, F_{\eta}$ are the distribution functions of random variables $\xi$ and $\eta$.) Our main goal is to get inequalities of the type (1.3) below which give upper bounds (continuity estimates) of the uniform distance $\rho(Z, \widetilde{Z})$ between the distributions of $Z$ and $\widetilde{Z}$ :

$$
\rho(Z, \widetilde{Z}) \leq c \max \left\{\rho\left(X_{1}, \widetilde{X}_{1}\right), \zeta_{2}\left(X_{1}, \widetilde{X}_{1}\right)\right\}
$$

Here $\zeta_{2}$ is Zolotarev's metric (see for instance, [11,13]) and $c$ is some constant.

In the particular case, when $p_{k}=q(1-q)^{k-1}, k=1,2, \ldots$ the random variables $Z, \widetilde{Z}$ in (1.1) and (1.2) are called geometric sums. The quantitative solution of the continuity (stability) problem for geometric sums (e.g. the solution given by (1.3)) provides a tool to estimate the stability of a number of stochastic models involving geometric sums. To give a hint about the importance of the problem, we first quote from [8]: "Despite the simplicity (or owing to this) such a sum can model many phenomena in insurance, queuing, finances, reliability, biology, storage and other real world fields". Second note that in an overwhelming majority of such applied models, one has to rely only on some approximations, operating with input data obtained from statistical estimations and (or) theoretical simplifications. In particular, one can face the situation where the distribution function of $\sum_{k=1}^{\nu} \widetilde{X}_{k}$ is handled to approximate a "real" and hence desired, but unknown (unavailable) distribution function of $\sum_{k=1}^{\nu} X_{k}$.

There are many publications on the theory and applications of geometric sums (or on "geometric convolutions", which are distributions of such sums). We confine ourselves to mentioning works [1-9], where geometric sums and their continuity play a decisive role.

In the case of geometric sums, inequality (1.3) supplies the uniform (with respect to $q \in(0,1))$ estimate of the nearness of $F_{Z}$ and $F_{\widetilde{Z}}$ measured in the uniform metric. In [8] the following bound has been obtained:

$$
\sup _{q \in(0,1)} \rho(Z, \widetilde{Z}) \leq \frac{2 M \sqrt{\delta}}{\sqrt{4 M+\delta}-\sqrt{\delta}}
$$

where $\delta:=\rho\left(X_{1}, \widetilde{X}_{1}\right), E X_{1}=E \widetilde{X}_{1}, M \geq \max \left\{E X_{1}^{2}, E \widetilde{X}_{1}^{2}\right\}$.

In inequality (1.4) the right-hand side is of order $\delta^{1 / 2}$ as $\delta \rightarrow 0$. In the present paper we restrict the class of random variables dealt with, assuming some "smoothness" properties of distributions of $X_{1}$ and $\widetilde{X}_{1}$. As a gain we obtain the first power of the distance

$$
\max \left\{\rho\left(X_{1}, \widetilde{X}_{1}\right), \zeta_{2}\left(X_{1}, \widetilde{X}_{1}\right)\right\}
$$

on the right hand side in (1.3) by making use of methods quite different from those in the book [8]. The technique of the present paper rather relates to that of [4, 5], where we studied the continuity of sums with respect to the total variation metric.

\section{Results and Discussion}

We will prove two version of inequality (1.3), each of them holding under different sets of assumptions. Such duplication is relevant since both versions have certain disadvantages, yet in some sense supplement each other. The main Theorems 1 and 2 are, in fact, direct consequences of upper bounds (2.8) and (2.9) for the uniform distance between sums of i.i.d. random variables. These bounds are of interest in contexts broader than that of the continuity analysis of geometric sums. 
Throughout this paper we use the following probability metrics $(X, Y$ are arbitrary random variables and $F_{X}, F_{Y}$ are their distribution functions):

(a) The uniform (Kolmogorov) metric:

$$
\rho(X, Y):=\sup _{x \in \mathbb{R}}\left|F_{X}(x)-F_{Y}(x)\right| .
$$

(b) The total variation metric:

$$
\mathbf{V}(X, Y):=\sup 2\{|P(X \in B)-P(Y \in B)|\}: B \text { are Borel subsets of } \mathbb{R}\} .
$$

Note that

$$
\mathbf{V}(X, Y)=\int_{-\infty}^{\infty}\left|f_{X}(x)-f_{Y}(x)\right| d x
$$

if the random variables have densities $f_{X}, f_{Y}$.

(c) Second order Zolotarev's metric:

$$
\zeta_{2}(X, Y):=\sup \left\{|E \varphi(X)-E \varphi(Y)|: \varphi \in \mathcal{D}_{2}\right\},
$$

where $\mathcal{D}_{2}$ denotes the set of all functions $\varphi: \mathbb{R} \rightarrow \mathbb{R}$ having (almost everywhere) second derivatives bounded by 1 .

(d) The maximum of the uniform and Zolotarev's metrics:

$$
\mu(X, Y):=\max \left\{\rho(X, Y), \zeta_{2}(X, Y)\right\} .
$$

It is well known (see for instance $[9,11]$ ) that the convergence in any above metric yields the weak convergence of random variables. Also, under the condition $E X=E Y$; $E X^{2}, E Y^{2}<\infty$, the distance $\zeta_{2}(X, Y)$ is finite. The integral representation for $\zeta_{2}$ is known (see $[9,11])$ :

$$
\zeta_{2}(X, Y)=\int_{-\infty}^{\infty}\left|\int_{-\infty}^{x}\left[F_{X}(t)-F_{Y}(t)\right] d t\right| d x
$$

In what follows we adopt the notations:

(i) $\left\{X_{1}, X_{2}, \ldots\right\},\left\{\widetilde{X}_{1}, \widetilde{X}_{2}, \ldots\right\}$ are two sequences of i.i.d. random variables;

(ii) $\quad \nu$ is a random variable assuming natural values and independent of

$$
\left\{X_{1}, X_{2}, \ldots\right\} \text { and }\left\{\widetilde{X}_{1}, \widetilde{X}_{2}, \ldots\right\}
$$

(iii) $Z=\sum_{k=1}^{\nu} X_{k}, \widetilde{Z}=\sum_{k=1}^{\nu}, \widetilde{X}_{k}$.

(iv) $\varphi(x)=\frac{1}{\sqrt{2 \pi}} e^{-x^{2} / 2}$ is the density of the standard normal distribution.

All results of this paper are proved using the following basic hypotheses, which we will not specially mention in the formulations of theorems.

Assumption B: $E X_{1}=E \widetilde{X}_{1}=: a$.

Using the notations: $\sigma^{2}=\operatorname{Var}\left(X_{1}\right), \widetilde{\sigma}^{2}=\operatorname{Var}\left(\widetilde{X}_{1}\right)$ we assume that $\sigma>0, \widetilde{\sigma}>0$ and also $E\left|X_{1}\right|^{3}<\infty, E\left|\widetilde{X}_{1}\right|^{3}<\infty$.

We exploit Assumption 1 in Theorems 1 and 3. On the other hand, we make use of Assumption 2 to prove Theorems 2 and 4. 
Assumption 1: Let $s \geq 1$ be fixed and $X=\sum_{k=1}^{s} X_{k}, \widetilde{X}=\sum_{k=1}^{s} \widetilde{X}_{k}$. We assume that both random variables $X$ and $\tilde{X}$ have bounded absolutely continuous densities denoted by $f$ and $\widetilde{f}$, respectively. Their derivatives $f^{\prime}, \widetilde{f}^{\prime}$ are bounded and belong to $\mathbb{L}_{1}(\mathbb{R})$.

Remark 1: We admit $s>1$ in Assumption 1 in order not to take out of consideration such important densities as the uniform densities, the exponential ones, some $\Gamma$-densities, etc.

Assumption 2: Let $\eta$ and $\widetilde{\eta}$ be normally distributed random variables with parameters $a, \sigma$ and $a, \widetilde{\sigma}$, respectively. We assume that for some $\alpha<1$ the following inequalities hold:

(a) $\max \left\{\mathbf{V}\left(X_{1}, \eta\right), \zeta_{2}\left(X_{1}, \eta\right)\right\} \leq \alpha(2 b)^{-1}$;

(b) $\max \left\{\mathbf{V}\left(\widetilde{X}_{1}, \widetilde{\eta}\right), \zeta_{2}\left(\widetilde{X}_{1}, \widetilde{\eta}\right)\right\} \leq \alpha(2 \widetilde{b})^{-1}$, where $b=\max \left\{1,6 r / \sigma^{2}\right\}, \widetilde{b}=$ $\max \left\{1,6 r / \widetilde{\sigma}^{2}\right\}$,

$$
r=\int_{-\infty}^{\infty}\left|\varphi^{\prime \prime}(x)\right| d x \approx 0.967883<1 .
$$

The metrics $\mathbf{V}$ and $\zeta_{2}$ were defined in (2.2) and (2.3), respectively.

We now present our results.

Theorem 1: Suppose that random variables $X_{1}$ and $\widetilde{X}_{1}$ satisfy Assumption 1 . Then

$$
\rho(Z, \widetilde{Z}) \leq \max \left\{2 s-1, c_{1}\right\} \mu\left(X_{1}, \widetilde{X}_{1}\right)
$$

where $c_{1}$ is some constant and the metrics $\rho$ and $\mu$ were defined in (2.1) and (2.4).

Theorem 2: Suppose that random variables $X_{1}$ and $\widetilde{X}_{1}$ satisfy Assumption 2 . Then

$$
\rho(Z, \widetilde{Z}) \leq \max \left\{1, c_{2}\right\} \mu\left(X_{1}, \widetilde{X}_{1}\right),
$$

where $c_{2}=\frac{1}{1-\alpha} \gamma\left(2 / \sigma^{2}+1 / \widetilde{\sigma}^{2}\right), \gamma=\sup _{x}\left|\varphi^{\prime}(x)\right| \approx 0.241971<0.242$.

In the next two theorems we use the notations:

$$
S_{n}=X_{1}+\ldots+X_{n}, \widetilde{S}_{n}=\widetilde{X}_{1}+\ldots+\widetilde{X}_{n}, n \geq 1 .
$$

Theorem 3: Under Assumption 1, we get, for every $n \geq 2 s$ :

$$
\rho\left(S_{n}, \widetilde{S}_{n}\right) \leq c_{1} \zeta_{2}\left(X_{1}, \widetilde{X}_{1}\right)
$$

with the same constant $c_{1}$ as in (2.6).

Theorem 4: Under Assumption 2, we get, for every $n \geq 1$ :

$$
\rho\left(S_{n}, \widetilde{S}_{n}\right) \leq \max \left\{1, c_{2}\right\} \mu\left(X_{1}, \widetilde{X}_{1}\right)
$$

with the same constant $c_{2}$ as in (2.7).

To start a discussion about the results obtained, we observe that a noteworthy feature of inequalities (2.7), (2.9) is the explicit form of the constants $c_{2}$ and $b, \widetilde{b}$, which depend only on variances on random variables $X_{1}$ and $\widetilde{X}_{1}$ (in Theorems 2, 4 and Assumption 2). To get upper bounds for these constants one needs to know only some lower bounds for variances $\sigma^{2}$ and $\widetilde{\sigma}^{2}$.

In contrast to such a good property, the constant $c_{1}$ in inequalities (2.6), (2.8) of Theorems 1 and 3 are far from being explicit and moreover, $c_{1}$ depends on certain characteristics of the densities $f$ and $\widetilde{f}$ of random variables $X$ and $\widetilde{X}$ defined in Assumption 1. As we will see in the proof of Theorem 3, 


$$
c_{1}=\frac{\left(1+s^{-1}\right) d}{\sigma^{2}}+\frac{\widetilde{d}}{\widetilde{\sigma}^{2}}
$$

where again, $\sigma^{2}=\operatorname{Var}\left(X_{1}\right), \widetilde{\sigma}^{2}=\operatorname{Var}\left(\widetilde{X}_{1}\right)$ and

$$
d=\sup _{k \geq s} \sup _{x}\left|f_{k}^{\prime}(x)\right|, \widetilde{d}=\sup _{k \geq s} \sup _{x}\left|\widetilde{f}_{k}^{\prime}(x)\right|
$$

where $f_{k}, \widetilde{f}_{k}$ denote, respectively, the densities of the following "normed sums"

$$
\frac{X_{1}+\ldots+X_{k}}{\sigma \sqrt{k}}, \frac{\widetilde{X}_{1}+\ldots+\widetilde{X}_{k}}{\widetilde{\sigma} \sqrt{k}}, k \geq s .
$$

Nevertheless, the proof of Theorem 3 ensures finiteness of the constants $d$ and $\widetilde{d}$ in (2.11) and displays the following asymptotics:

$$
\lim _{k \rightarrow \infty} \sup _{x}\left|f_{k}^{\prime}(x)\right| \overline{\bar{k}} \lim _{\rightarrow \infty} \sup _{x}\left|\widetilde{f}_{k}^{\prime}(x)\right|=\sup _{x}\left|\varphi^{\prime}(x)\right|=\gamma \approx 0.242,
$$

where again, $\varphi(x)=\frac{1}{\sqrt{2 \pi}} e^{-x^{2} / 2}, x \in \mathbb{R}$. Using the explicit forms of densities of sums and computer calculations, it is not a difficult task to estimate the constants $d$ and $\widetilde{d}$ from (2.11) in a number of important particular cases. For example, we obtain the following results. (Note, that the values of $d$ and $\widetilde{d}$ do not depend on expectations):

(a) $\quad d<0.242$ if $X_{1}$ is a normally distributed random variable (with arbitrary parameters, taking $s=1$ );

(b) $\quad d=2$ if $X_{1}$ is an exponentially distributed random variable (with arbitrary parameter, taking $s=2$ );

(c) $\quad d=0.25$ if $X_{1}$ is a uniformly distributed random variable (with arbitrary parameters, taking $s=2$ );

(d) $\quad d=2$ if $X_{1}$ has the $\Gamma$-density with parameter $\alpha=2$; and arbitrary parameter $\lambda$ (taking $s=1$ );

(e) $\quad d<0.523$ if $X_{1}$ has the $\Gamma$-density with parameter $\alpha=4$; and arbitrary parameter $\lambda$ (taking $s=1$ ).

It is instructive to trace out the behavior of the value $\beta_{k}=\sup _{x \in \mathbb{R}}\left|f_{k}^{\prime}(x)\right|$ as a func-

tion of $k$ in the particular cases. The next four tables and accompanying picture give a eyewitness account of such behavior.

Table 1: The Case of the Exponential Density

$\begin{array}{lllllllll}k & 2 & 3 & 4 & 5 & 6 & 7 & 8 & 9 \\ \beta_{k} & 2 & .6913 & .5220 & .4511 & .4109 & .3857 & .3678 & .3542 \\ k & 10 & 11 & 12 & 15 & 20 & 30 & 50 & 80 \\ \beta_{k} & .3442 & .3359 & .3290 & .3145 & .2997 & .2852 & .2726 & .2647\end{array}$


Table 2: The Case of the Uniform Density

$\begin{array}{llllllcll}k & 2 & 3 & 4 & 5 & 6 & 7 & 8 & 9 \\ \beta_{k} & .1667 & .2500 & .2222 & .2273 & .2300 & .2316 & .2329 & .2339 \\ & & & & & & & & \\ k & 10 & 11 & 12 & 15 & 20 & 30 & 50 & 80 \\ \beta_{k} & .2344 & .2353 & .2357 & .2370 & .2383 & .2385 & .2404 & .2414\end{array}$

Table 3: The Case of the $\Gamma$-Density with $\alpha=2$

$\begin{array}{llllllllll}k & 1 & 2 & 3 & 4 & 5 & 6 & 7 & 8 & 9 \\ \beta_{k} & 2 & .5220 & .4109 & .3678 & .3442 & .3290 & .3186 & .3107 & .3047 \\ k & 10 & 11 & 12 & 15 & 20 & 30 & 50 & 80 & 100 \\ \beta_{k} & .2997 & .2960 & .2926 & .2852 & .2773 & .2690 & .2618 & .2568 & .2550\end{array}$

Table 4: The Case of the $\Gamma$-Density with $\alpha=4$

$\begin{array}{llllllllll}k & 1 & 2 & 3 & 4 & 5 & 6 & 7 & 8 & 9 \\ \beta_{k} & .5224 & .3678 & .3290 & .3107 & .2997 & .2926 & .2872 & .2833 & .2801 \\ k & 10 & 11 & 12 & 15 & 20 & 30 & 50 & 80 & 100 \\ \beta_{k} & .2773 & .2753 & .2734 & .2690 & .2647 & .2597 & .2550 & .2518 & .2503\end{array}$

Below we present the graphs of absolute values of the derivatives $f_{k}^{\prime}(x)$ calculated for $k=1,2, \ldots, 20$ with $X_{1}$ having the $\Gamma$-density with $\alpha=4, \lambda=2$.

The above tables show that, at least in these examples, the sequence $\left.\sup _{x \in \mathbb{R}}\left|f_{k}^{\prime}(x)\right|, k \geq s\right\}$ approaches the limit $\gamma \approx 0.241971$ "in a rather regular way".

This allows us to estimate easily the value $d$ in (2.11).

Figure 1: The Graphs of $\left|f_{k}^{\prime}(x)\right|$, for $k=1,2, \ldots, 20$. 
We can also offer a rough upper bound of the constant $d$ when we have some information on the characteristic function $\varphi(t)$ of the random variable $X_{1} / \sigma$.

Assume that some $\delta, 0<\delta<1$, is known such that

$$
|\varphi(t)| \leq \frac{\delta}{|t|^{3 / s}} \text { for }|t| \geq 1
$$

Now, for $k \geq s$ let $\varphi_{k}$ denote the characteristic function of the random variable $\frac{X_{1}+\ldots+X_{k}}{\sigma \sqrt{k}}$ and let $f_{k}$ be the density of this random variables.

From (2.12) it follows that $\int_{-\infty}^{\infty}|t| \quad\left|\varphi_{k}(t)\right| d t<\infty$ and hence we can calculate $f_{k}^{\prime}$ as follows

$$
f_{k}^{\prime}(x)=\frac{1}{2 \pi} \int_{-\infty}^{\infty}(-i t) e^{-i t x} \varphi_{k}(t) d t, x \in \mathbb{R}
$$

Thus

$$
\begin{gathered}
\sup _{x}\left|f_{k}^{\prime}(x)\right| \leq \frac{1}{2 \pi} \int_{-\infty}^{\infty}|t|\left|\varphi_{k}(t)\right| d t=\frac{k}{2 \pi} \int_{-\infty}^{\infty}|t||\varphi(t)|{ }^{k} d t \\
=\frac{k}{2 \pi} \int_{-1}^{1}|t||\varphi(t)|^{k} d t+\frac{k}{2 \pi} \int_{|t| \geq 1}|t||\varphi(t)|{ }^{k} d t .
\end{gathered}
$$

By (2.12) the second integral in (2.13) is less than $\int_{|t| \geq 1}|t| \frac{\delta^{k}}{|t|^{\frac{3 k}{s}}} d t=\frac{2 \delta^{k} s}{3 k-2 s}$ and for this, the second term on the right-hand side of (2.13) is smaller than $\frac{s}{\pi} \frac{k \delta^{k}}{3 k-2 s} \leq \frac{s \delta^{s}}{\pi}$ (since the function $\frac{x \delta^{x}}{3 x-2 s}$ is decreasing for $x \geq s$ ).

Applying Theorem 1, Ch. 1 in [10], we see that (2.12) yields the following inequality:

$$
|\varphi(t)| \leq 1-\frac{1-\delta^{2}}{8} t^{2} \text { for }|t|<1
$$
have:

The last inequality allows us to estimate the first term on the right-hand side of (2.13). We

$$
\begin{gathered}
\frac{k}{2 \pi} \int_{-1}^{1}|t||\varphi(t)|{ }^{k} d t \leq \frac{k}{\pi} \int_{0}^{1} t\left[1-\left(\frac{1-\delta^{2}}{8}\right) t^{2}\right]^{k} d t \\
=\frac{4}{\left(1-\delta^{2}\right) \pi} \frac{k}{k+1}\left[1-\left(1-\frac{1-\delta^{2}}{8}\right)^{k+1}\right] \\
\leq \frac{4}{\left(1-\delta^{2}\right) \pi} .
\end{gathered}
$$

Gathering all above inequalities together we finally get the estimate: 


$$
d=\sup _{k \geq s} \sup _{x}\left|f_{k}^{\prime}(x)\right| \leq \frac{1}{\pi}\left[s \delta^{s}+\frac{4}{1-\delta^{2}}\right]
$$

Let us now turn to inequalities (2.7), (2.9) in Theorems 2, 4. Everything is all right with the constant $c_{2}$ in these inequalities but the requirements $(a)$ and $(b)$ of Assumption 2 (which demand the nearness of random variables to normal ones) is undesirable. This is evidently a weak point that reduces the range of possible applications. For this, Theorems 2 and 4 should be considered as a first step towards better results of this kind. Note that normally distributed random variables have been chosen in Assumption 2 only to fix the idea. The normal density can be replaced by the uniform one, $\Gamma$-density with $\alpha \geq 1$ and more generally by any density which satisfies the hypotheses of Lemma 1 in [5] and for which the constant in this lemma can be effectively calculated. We reproduce here the formulation of this lemma. (See Lemma 2 in the next section).

The next remark outlines an even more promising way to overcome restrictions $(a)$ and (b) in Assumption 2.

Remark 2: The revision of the proof of Lemma 2 of the next section shows that inequalities (3.4) and (3.5) can be replaced by the following ones: for $n \geq 2 s$

$$
\mathbf{V}\left(S_{n}, Y_{n}\right) \leq \bar{b} \Delta_{s}
$$

provided that

$$
\begin{aligned}
& \Delta_{s}:=\max \left\{\mathbf{V}\left(\frac{X_{1}+\ldots+X_{s}}{\sqrt{s}}, \frac{\xi_{1}+\ldots+\xi_{s}}{\sqrt{s}}\right),\right. \\
& \left.\zeta_{2}\left(\frac{X_{1} \ldots+X_{s}}{\sqrt{s}}, \frac{\xi_{1}+\ldots+\xi_{s}}{\sqrt{s}}\right)\right\} \leq(2 \bar{b})^{-1}
\end{aligned}
$$

where $s \geq 1$ is an arbitrary, but fixed integer and the explicitly calculated constant $\bar{b}$ does not depend on $s$. The point is to replace conditions $(a)$ and $(b)$ in Assumption 2 by the conditions:

$$
\begin{gathered}
\max \left\{\mathbf{V}\left(\frac{X_{1}+\ldots+X_{s}}{\sqrt{s}}, \eta\right), \zeta_{2}\left(\frac{X_{1}+\ldots+X_{s}}{\sqrt{s}}, \eta\right)\right\} \leq \alpha(2 \bar{b})^{-1} \\
\max \left\{\mathbf{V}\left(\frac{\widetilde{X}_{1}+\ldots+\widetilde{X}_{s}}{\sqrt{s}}, \widetilde{\eta}\right), \zeta_{2}\left(\frac{\widetilde{X}_{1}+\ldots+\widetilde{X}_{s}}{\sqrt{s}}, \widetilde{\eta}\right)\right\} \leq \alpha(2 \widetilde{b})^{-1}
\end{gathered}
$$

and to take an advantage of known estimates of the rate of convergence in the central limit theorem in order to choose $s$ in (2.14), (2.15) so that these inequalities are satisfied for a wide enough class of random variables $X_{1}, \widetilde{X}_{1}$. (In (2.14), (2.15) $\eta, \widetilde{\eta}$

are, of course, normal random variables with corresponding parameters.)

For instance, to estimate $\mathbf{V}\left(\frac{X_{1}+\ldots+X_{s}}{\sqrt{s}}, \eta\right)$, one can exploit the following result given in [10], Ch. VII, Supplement. Suppose a random variable $X_{1}$ has a density bounded by a constant $K$. Denoting by $p_{n}$ the density of $\frac{X_{1}+\ldots+X_{n}-n a}{\sigma \sqrt{n}}$, one has for every $x \in \mathbb{R}$ :

$$
\left|p_{n}(x)-\frac{1}{\sqrt{2 \pi}} e^{-x^{2} / 2}\right| \leq \frac{A\left\{E\left|X_{1}\right|^{3}\right\}^{3} \max \left\{1, K^{5}\right\}}{\sqrt{n}\left(1+|x|^{2}\right) \sigma^{9}}
$$


where $A$ is an absolute constant. Integrating this inequality one gets the upper bound for

$$
\mathbf{V}\left(\frac{X_{1}+\ldots+X_{n}}{\sqrt{n}}, \eta\right)
$$

\section{Auxiliary Results and the Proofs of the Theorems}

The proof of Theorem 3 makes use of the following simple assertion.

Lemma 1: Let $X, Y$ and $Z$ be independent random variables such that:

(a) $E X=E Y ; E X^{2}, E Y^{2}<\infty$;

(b) the distribution function $F_{Z}$ has a bounded, absolutely continuous density $f_{Z}$;

(c) the derivative $f_{Z}^{\prime}$ is a bounded function (on a set where it exists).

Then

$$
\rho(X+Z, Y+Z) \leq \sup _{x}\left|f_{Z}^{\prime}(x)\right| \zeta_{2}(X, Y) .
$$

Proof: By the definitions of $\rho$ and of convolution we get

$$
\begin{aligned}
& \rho(X+Z, Y+Z)=\sup _{x \in \mathbb{R}}\left|F_{X+Z}(x)-F_{Y+Z}(x)\right| \\
& \quad=\sup _{x \in \mathbb{R}}\left|\int_{-\infty}^{\infty} F_{Z}(x-t) d\left[F_{X}(t)-F_{Y}(t)\right]\right| .
\end{aligned}
$$

Integration by parts in

$$
I(x)=\int_{-\infty}^{\infty} F_{Z}(x-t) d\left[F_{X}(t)-F_{Y}(t)\right]
$$

gives

$$
I(x)=\int_{-\infty}^{\infty} f_{Z}(x-t)\left[F_{X}(t)-F_{Y}(t)\right] d t
$$

Again, integrating by parts, we get

$$
\begin{aligned}
& I(x)=\int_{-\infty}^{\infty} f_{Z}(x-t) d \int_{-\infty}^{t}\left[F_{X}(s)-F_{Y}(s)\right] d s \\
& =\int_{-\infty}^{\infty} f_{Z}^{\prime}(x-t)\left[\int_{-\infty}^{t}\left[F_{X}(s)-F_{Y}(s)\right] d s\right] d t
\end{aligned}
$$


(Recall $E X=E Y$, hence $\lim _{t \rightarrow \infty} \int_{-\infty}^{t}\left[F_{X}(s)-F_{Y}(s)\right] d s=0$ ). From (3.1) and (3.2) we obtain the following inequality

$$
\begin{gathered}
\rho(X+Z, Y+Z) \leq \sup _{x \in \mathbb{R} \infty} \int_{\mathbb{R}}^{\infty}\left|f_{Z}^{\prime}(x-t)\right|\left|\int_{-\infty}^{t}\left[F_{X}(s)-F_{Y}(s)\right] d s\right| d t \\
\quad \leq \sup _{x \in \mathbb{R}}\left|f_{Z}^{\prime}(x)\right| \int_{-\infty}^{\infty}\left|\int_{-\infty}^{t}\left[F_{X}(s)-F_{Y}(s)\right] d s\right| d t .
\end{gathered}
$$

The second (integral) factor in (3.3) is the integral representation (2.5) of Zolotarev's metric.

The following Lemma 2 appeared in [5] and was proven there (see Lemma 1 in [5] and related Theorem 3 in [12]). Lemma 3 was given as Lemma 1 in [12]. We will use both these facts in the proof of Theorem 4.

Lemma 2: Let $\xi_{1}, \xi_{2}, \ldots$ i.i.d. random variables such that $E\left(\xi_{1}\right)=E\left(X_{1}\right), E \xi_{1}^{2}<\infty$ and $Y_{n}=\xi_{1}+\ldots+\xi_{n}, S_{n}=X_{1}+\ldots+X_{n}, n \geq 1$.

Assume that the random variable $\xi_{1}$ has a bounded density $p$ satisfying the following conditions:

(a) the derivative $p^{\prime}$ is bounded and absolutely continuous;

(b) the derivatives $p^{\prime}$, $p^{\prime \prime}$ belong to $\mathbb{L}_{1}(\mathbb{R})$;

(c) for some $\theta>0$

$$
\int_{|x|>\theta n}\left|p^{\prime \prime}(x)\right| d x=O\left(n^{-1}\right) \text { as } n \rightarrow \infty .
$$

Then there exist a constant $b$, independent of the distribution of $X_{1}$, such that for $n \geq 1$

$$
\mathbf{V}\left(S_{n}, Y_{n}\right) \leq b \Delta
$$

provided that

$$
\Delta:=\max \left\{\mathbf{V}\left(X_{1}, \xi_{1}\right), \zeta_{2}\left(X_{1}, \xi_{1}\right)\right\} \leq(2 b)^{-1} .
$$

Remark 3: As shown in [5], the constant $b$ in (3.4) and (3.5) is expressed in the form:

$$
\begin{gathered}
b=\max \left\{1,6 r / \sigma_{0}^{2}\right\}, \sigma_{0}^{2}=\operatorname{Var}\left(\xi_{1}\right), \\
r=\sup _{k \geq 1 \infty} \int_{t}^{\infty}\left|f_{k}^{\prime \prime}(x)\right| d x<\infty,
\end{gathered}
$$

where $f_{k}$ is the density of the random variable $\frac{\xi_{1}+\ldots+\xi_{k}}{\sigma_{0} \sqrt{k}}, k=1,2, \ldots$.

Note that for normally distributed random variables $\xi_{1}, \xi_{2}, \ldots$ we have $f_{k}=\varphi$ and easily calculate $r \approx 0.967883<1$.

Lemma 3: Let $X, Y, Z$ and $T$ be independent random variables.

Then 


$$
\rho(X+Z, Y+Z) \leq \rho(X, Y) \boldsymbol{V}(Z, T)+\rho(X+T, Y+T) .
$$

The Proofs of Theorem 1 and 2: By the definition of the uniform metric we get (applying the total probability formula):

$$
\begin{gathered}
\rho(Z, \widetilde{Z})=\sup _{x \in \mathbb{R}}\left|P\left(\sum_{k=1}^{\nu} X_{k} \leq x\right)-P\left(\sum_{k=1}^{\nu} \widetilde{X}_{k} \leq x\right)\right| \\
=\sup _{x \in \mathbb{R}}\left|\sum_{n=1}^{\infty}\left[P\left(\sum_{k=1}^{n} X_{k} \leq x\right)-P\left(\sum_{k=1}^{n} \widetilde{X}_{k} \leq x\right)\right] P(\nu=n)\right| \\
\leq \sum_{n=1}^{\infty} \sup _{x \in \mathbb{R}}\left|P\left(\sum_{k=1}^{n} X_{k} \leq x\right)-P\left(\sum_{k=1}^{n} \widetilde{X}_{k} \leq x\right)\right| P(\nu=n) \\
=\sum_{n=1}^{\infty} \rho\left(S_{n}, \widetilde{S}_{n}\right) P(\nu=n) .
\end{gathered}
$$

Having applied the well-known regularity property of $\rho$, we write for $n=1, \ldots, 2 s-1$ $(s=1$ in Theorem 2):

$$
\begin{gathered}
\rho\left(S_{n}, \widetilde{S}_{n}\right)=\rho\left(X_{1}+\ldots+X_{n}, \widetilde{X}_{1}+\ldots+\widetilde{X}_{n}\right) \\
\leq n \rho\left(X_{1}, \widetilde{X}_{1}\right) \leq(2 s-1) \mu\left(X_{1}, \widetilde{X}_{1}\right) .
\end{gathered}
$$

On the other hand, for $n \geq 2 s$, due to Theorems 3 and 4 we can write the corresponding inequalities as follows:

$$
\begin{gathered}
\rho\left(S_{n}, \widetilde{S}_{n}\right) \leq c_{1} \mu\left(X_{1}, \widetilde{X}_{1}\right), \\
\rho\left(S_{n}, \widetilde{S}_{n}\right) \leq \max \left\{1, c_{2}\right\} \mu\left(X_{1}, \widetilde{X}_{1}\right) .
\end{gathered}
$$

Combining inequalities (3.7), (3.8) and (3.9), we complete the proofs of the theorems.

The Proof of Theorem 3: Let $n \geq 2 s$ be an arbitrary, but fixed integer and let $m=[n / 2]$ ( $[x]$ stands for the integer part of $x$ ).

We denote:

$$
\begin{gathered}
Z_{m}=X_{1}+\ldots+X_{m}, \quad Z_{m}^{\prime}=X_{m+1}+\ldots+X_{n} \\
\widetilde{Z}_{m}=\widetilde{X}_{1}+\ldots+\widetilde{X}_{m}, \widetilde{Z}_{m}^{\prime}=\widetilde{X}_{m+1}+\ldots+\widetilde{X}_{n} .
\end{gathered}
$$

By the triangle inequality:

$$
\rho\left(S_{n}, \widetilde{S}_{n}\right) \leq \rho\left(Z_{m}+Z_{m}^{\prime}, Z_{m}+\widetilde{Z}_{m}^{\prime}\right)+\rho\left(Z_{m}+\widetilde{Z}_{m}^{\prime}, \widetilde{Z}_{m}+\widetilde{Z}_{m}^{\prime}\right) .
$$

Applying Lemma 1 and the following property of the uniform metric $\rho(a X, a Y)=\rho(X, Y)$, $a \neq 0$, we get 


$$
\begin{gathered}
\rho\left(Z_{m}+Z_{m}^{\prime}, Z_{m}+\widetilde{Z}_{m}^{\prime}\right)=\rho\left(\frac{Z_{m}}{\sigma \sqrt{m}}+\frac{Z_{m}^{\prime}}{\sigma \sqrt{m}}, \frac{Z_{m}}{\sigma \sqrt{m}}+\frac{\widetilde{Z}_{m}^{\prime}}{\sigma \sqrt{m}}\right) \\
\leq \sup _{x}\left|f_{\frac{Z_{m}}{\sigma \sqrt{m}}}^{\prime}(x)\right| \zeta_{2}\left(\frac{Z_{m}^{\prime}}{\sigma \sqrt{m}}, \frac{\widetilde{Z}_{m}^{\prime}}{\sigma \sqrt{m}}\right) .
\end{gathered}
$$

The "ideal properties" of Zolotarev's metric:

$$
\zeta_{2}\left(a \sum_{i=1}^{n} \xi_{i}, a \sum_{i=1}^{n} \eta_{i}\right) \leq a^{2} \sum_{i=1}^{n} \zeta_{2}\left(\xi, \eta_{i}\right),
$$

( $\xi_{i}$ and $\eta_{i}$ are independent) (see, for instance, $[9,11,13]$ ) yield the following chain of inequalities:

$$
\begin{gathered}
\zeta_{2}\left(\frac{Z_{m}^{\prime}}{\sigma \sqrt{m}}, \frac{\widetilde{Z}_{m}^{\prime}}{\sigma \sqrt{m}}\right) \leq \frac{1}{\sigma^{2} m} \sum_{k=m+1}^{n} \zeta_{2}\left(X_{k}, \widetilde{X}_{k}\right) \leq \frac{n-[n / 2]}{\sigma^{2}[n / 2]} \zeta_{2}\left(X_{1}, \widetilde{X}_{1}\right) \\
\leq \frac{\left(1+s^{-1}\right)}{\sigma^{2}} \zeta_{2}\left(X_{1}, \widetilde{X}_{1}\right)
\end{gathered}
$$

since

$$
\sup _{n \geq s}(n-[n / 2]) /[n / 2]=\left(1+s^{-1}\right) .
$$

By the same arguments we get

$$
\begin{gathered}
\rho\left(Z_{m}+\widetilde{Z}_{m}^{\prime}, \widetilde{Z}_{m}+\widetilde{Z}_{m}^{\prime}\right)=\rho\left(\frac{Z_{m}}{\widetilde{\sigma} \sqrt{n-m}}+\frac{\widetilde{Z}_{m}^{\prime}}{\widetilde{\sigma} \sqrt{n-m}}, \frac{\widetilde{Z}_{m}}{\widetilde{\sigma} \sqrt{n-m}}+\frac{\widetilde{Z}_{m}^{\prime}}{\widetilde{\sigma} \sqrt{n-m}}\right) \\
\leq \sup _{x}\left|f_{\frac{Z_{m}^{\prime}}{\widetilde{\sigma} \sqrt{n-m}}}^{\prime}(x)\right| \frac{1}{\widetilde{\sigma}^{2}} \frac{[n / 2]}{n-[n / 2]} \zeta_{2}\left(X_{1}, \widetilde{X}_{1}\right) \\
\leq \sup _{x}\left|f_{\frac{\widetilde{Z}_{m}^{\prime}}{\widetilde{\sigma} \sqrt{n-m}}}^{\prime}(x)\right| \frac{1}{\widetilde{\sigma}^{2}} \zeta_{2}\left(X_{1}, \widetilde{X}_{1}\right) .
\end{gathered}
$$

From inequalities (3.11), (3.12) and (3.13) the desired result (2.8) follows with $c_{1}=\frac{\left(1+s^{-1}\right) d}{\sigma^{2}}+\frac{\widetilde{d}}{\widetilde{\sigma}^{2}}$, if we show that 


$$
d:=\sup _{k \geq s} \sup _{x}\left|f_{\frac{Z_{k}}{\sigma \sqrt{m}}}^{\prime}(x)\right|<\infty \text { and } \tilde{d}:=\sup _{k \geq s} \sup _{x}\left|f_{\frac{\widetilde{Z}_{k}}{\widetilde{\gamma} k}}^{\prime}(x)\right|<\infty \text {. }
$$

Let us verify (3.14), say, for densities $f_{k} \equiv f_{\frac{Z_{k}}{\sigma \sqrt{k}}}$ of the random variables $\frac{X_{1}+\ldots+X_{k}}{\sigma \sqrt{k}}$. First of all, note that

$$
\sup _{x}\left|f_{\frac{Z_{k}}{\sigma \sqrt{k}}}^{\prime}(x)\right|=\sup _{x}\left|f_{\frac{\bar{Z}_{k}}{\sigma \sqrt{k}}}^{\prime}(x)\right|
$$

where

$$
\bar{Z}_{k}=\left(X_{1}-E X_{1}\right)+\ldots+\left(X_{k}-E X_{k}\right)
$$

Denote

$$
\bar{f}_{k}:=f_{\frac{\bar{z}_{k}}{\sigma \sqrt{k}}} .
$$

The hypotheses of Theorem 3, particularly, the conditions $E\left|X_{1}\right|^{3}<\infty, f^{\prime} \in \mathbb{L}_{1}(\mathbb{R})$ guarantee the hypotheses of Theorem 7, Ch. VI in [10], which in our case states:

$$
\bar{f}_{k}^{\prime}(x)=\frac{1}{\sqrt{2 \pi}} \frac{d^{2}}{d x^{2}}\left[\int_{-\infty}^{x} e^{-y^{2} / 2} d y-e^{-x^{2} / 2} \frac{E X_{1}^{3}\left(x^{2}-1\right)}{6 \sigma^{3} \sqrt{k}}\right]+o\left(k^{-1 / 2}\right),
$$

uniformly in $x \in \mathbb{R}$.

The last equality implies finiteness of $d$ in (3.14). This also provides the following asymptotic behavior of $\max _{x}\left|f_{k}^{\prime}(x)\right|$ :

$$
\max _{x}\left|f_{k}^{\prime}(x)\right| \rightarrow \max _{x}\left|\left[\frac{1}{\sqrt{2 \pi}} e^{-x^{2} / 2}\right]^{\prime}\right| \text { as } k \rightarrow \infty .
$$

The Proof of Theorem 4: Let $\eta_{1}, \eta_{2}, \ldots$ and $\widetilde{\eta}_{1}, \widetilde{\eta}_{2}, \ldots$ be i.i.d. normally distributed random variables with parameters $a, \sigma$ and $a, \widetilde{\sigma}$ respectively. Set $Y_{k}=\eta_{1}+\ldots+\eta_{k}$; $\widetilde{Y}_{k}=\widetilde{\eta}_{1}+\ldots+\widetilde{\eta}_{k}, k=1,2, \ldots$

For $n \geq 2$ we introduce the same notation $Z_{m}, Z_{m}^{\prime} ; \widetilde{Z}_{m}, \widetilde{Z}_{m}^{\prime}$ as in relations (3.10).

By the triangle inequality and by virtue of inequality (3.6) in Lemma 3 we can write:

$$
\begin{gathered}
\rho\left(S_{n}, \widetilde{S}_{n}\right) \leq \rho\left(Z_{m}+Z_{m}^{\prime}, Z_{m}+\widetilde{Z}_{m}^{\prime}\right)+\rho\left(Z_{m}+\widetilde{Z}_{m}^{\prime}, \widetilde{Z}_{m}+\widetilde{Z}_{m}^{\prime}\right) \\
\leq \rho\left(Z_{m}^{\prime}, \widetilde{Z}_{m}^{\prime}\right) \mathbf{V}\left(Z_{m}, Y_{m}\right)+\rho\left(Z_{m}^{\prime}+Y_{m}, \widetilde{Z}_{m}^{\prime}+Y_{m}\right) \\
+\rho\left(Z_{m}, \widetilde{Z}_{m}\right) \mathbf{V}\left(\widetilde{Z}_{m}^{\prime}, \widetilde{Y}_{n-m}\right)+\rho\left(Z_{m}+\widetilde{Y}_{n-m}, \widetilde{Z}_{m}+\widetilde{Y}_{n-m}\right)
\end{gathered}
$$

From Assumption 2 and Lemma 2 it follows that 


$$
\mathbf{V}\left(Z_{m}, Y_{m}\right) \leq \alpha / 2, \mathbf{V}\left(\widetilde{Z}_{m}^{\prime}, \widetilde{Y}_{n-m}\right) \leq \alpha / 2 \text { for every } n \geq 2 .
$$

(Since in this case $\sigma_{0}=\sigma$ and random variables $\xi_{k}$ in Lemma 2 are normal; see Lemma 2 and Remark 3.)

Applying (as in the preceding proof) the suitable properties of metrics $\rho$ and $\zeta_{2}$, Lemma 1 and observing that $Y_{m} / \sigma \sqrt{m}$ are normal random variables of unit variance (and hence $\sup _{x}\left|f_{\frac{Y m}{\sigma \sqrt{m}}}^{\prime}(x)\right|=\sup _{x}\left|\varphi^{\prime}(x)\right|$, with $\varphi(x)=\frac{1}{\sqrt{2 \pi}} e^{-x^{2} / 2}$ ), we obtain the following inequalities:

$$
\begin{gathered}
\rho\left(Z_{m}^{\prime}+Y_{m}, \widetilde{Z}_{m}^{\prime}+Y_{m}\right) \\
=\rho\left(\frac{Z_{m}^{\prime}}{\sigma \sqrt{m}}+\frac{Y_{m}}{\sigma \sqrt{m}}, \frac{\widetilde{Z}_{m}^{\prime}}{\sigma \sqrt{m}}+\frac{Y_{m}}{\sigma \sqrt{m}}\right) \leq \sup _{x \in \mathbb{R}}\left|f_{\frac{Y_{m}}{\sigma \sqrt{m}}}^{\prime}(x)\right| \zeta_{2}\left(\frac{Z_{m}^{\prime}}{\sigma \sqrt{m}}, \frac{\widetilde{Z}_{m}^{\prime}}{\sigma \sqrt{m}}\right) \\
\quad \leq \sup _{x \in \mathbb{R}}\left|\varphi^{\prime}(x)\right| \frac{1}{m \sigma^{2}} \zeta_{2}\left(X_{m+1}+\ldots+X_{n}, \widetilde{X}_{m+1}+\ldots+\widetilde{X}_{n}\right) \\
\leq \frac{\gamma}{\sigma^{2}} \frac{n-[n / 2]}{[n / 2]} \zeta_{2}\left(X_{1}, \widetilde{X}_{1}\right) \leq \frac{2 \gamma}{\sigma^{2}} \zeta_{2}\left(X_{1}, \widetilde{X}_{1}\right) .
\end{gathered}
$$

(Here $\gamma: \sup _{x \in \mathbb{R}}\left|\varphi^{\prime}(x)\right|<0.242$ ).

Similarly,

$$
\begin{gathered}
\rho\left(Z_{m}+\widetilde{Y}_{n-m}, \widetilde{Z}_{m}+\widetilde{Y}_{n-m}\right) \leq \sup _{x \in \mathbb{R}}\left|f_{\frac{\widetilde{Y}_{n-m}}{\widetilde{\sigma} \sqrt{n-m}}}^{\prime}(x)\right| \zeta_{2}\left(\frac{Z_{m}}{\widetilde{\sigma} \sqrt{n-m}}, \frac{\widetilde{Z}_{m}}{\widetilde{\sigma} \sqrt{n-m}}\right) \\
\leq \frac{\gamma}{\widetilde{\sigma}^{2}} \frac{[n / 2]}{n-[n / 2]} \zeta_{2}\left(X_{1}, \widetilde{X}_{1}\right) \leq \frac{\gamma}{\widetilde{\sigma}^{2}} \zeta_{2}\left(X_{1}, \widetilde{X}_{1}\right) .
\end{gathered}
$$

Finally, from (3.15), (3.16), (3.17) and (3.18) we have

$$
\begin{gathered}
\rho\left(S_{n}, \widetilde{S}_{n}\right) \leq \frac{\alpha}{2} \rho\left(Z_{m}^{\prime}, \widetilde{Z}_{m}^{\prime}\right)+\frac{\alpha}{2} \rho\left(Z_{m}, \widetilde{Z}_{m}\right)+\left(\frac{2 \gamma}{\sigma^{2}}+\frac{\gamma}{\widetilde{\sigma}^{2}}\right) \zeta_{2}\left(X_{1}, \widetilde{X}_{1}\right) \\
\leq \alpha \max \left\{\mu\left(Z_{m}^{\prime}, \widetilde{Z}_{m}^{\prime}\right), \mu\left(Z_{m}, \widetilde{Z}_{m}\right)\right\}+\gamma\left(\frac{2}{\sigma^{2}}+\frac{1}{\widetilde{\sigma}^{2}}\right) \mu\left(X_{1}, \widetilde{X}_{1}\right) .
\end{gathered}
$$

To complete the proof we use induction arguments together with inequality (3.19). We have for $n=1, \rho\left(S_{n}, \widetilde{S}_{n}\right)=\rho\left(X_{1}, \widetilde{X}_{1}\right) \leq c \mu\left(X_{1}, \widetilde{X}_{1}\right)$ if $c \geq 1$.

Suppose that $\rho\left(S_{k}, \widetilde{S}_{k}\right) \leq c \mu\left(X_{1}, \widetilde{X}_{1}\right)$ for $k \leq n-1$.

From (3.19) it follows that

$$
\rho\left(S_{n}, \widetilde{S}_{n}\right) \leq\left[\alpha c+\gamma\left(\frac{2}{\sigma^{2}}+\frac{1}{\widetilde{\sigma}^{2}}\right)\right] \mu\left(X_{1}, \widetilde{X}_{1}\right)
$$


The induction would hold if

$$
\alpha c+\gamma\left(\frac{2}{\sigma^{2}}+\frac{1}{\widetilde{\sigma}^{2}}\right) \leq c,
$$

or if we choose the constant $c$ to satisfy the inequalities

$$
c \geq \frac{\gamma}{1-\alpha}\left(\frac{2}{\sigma^{2}}+\frac{1}{\widetilde{\sigma}^{2}}\right) \text {, and } c \geq 1 .
$$

\section{Acknowledgement}

The authors would like to thank the referee, the associate and technical editors for their valuable suggestions, which have improved the presentation of the paper.

\section{References}

[1] Asmussen, S., Applied Probability and Queues, J. Wiley \& Sons, Chichester, 1987.

[2] Brown, M., Error bounds for exponential approximation of geometric convolutions, The Annals of Prob. 18 (1990), 1388-1402.

[3] Gertsbakh, I., Asymptotic methods in reliability theory: A review, Adv. Appl. Prob. 16 (1984), 147-175.

[4] Gordienko, E.I., Estimates of stability of geometric convolutions, Appl. Math. Letters 12 (1999), 103-106.

[5] Gordienko, E.I. and Ruiz de Chávez, J., New estimates of continuity in M/GI/1/ $\infty$ queues, Queueing Systems 29 (1998), 175-188.

[6] Kalashnikov, V., Two-side estimates of geometric convolutions, Lect. Notes in Math. 1546 (1993), 76-88.

[7] Kalshnikov, V., Two-side estimates of ruin probabilities, Scand. Actuarial J. 1 (1996), $1-18$.

[8] Kalashnikov, V., Geometric Sums: Bounds for Rare Events with Applications, Kluwer Academic Publishers, Dordrecht 1997.

[9] Kalashnikov, V. and Rachev, S., Mathematical Methods for Construction of Queueing Models, Wadsworth and Brooks/Cole, Pacific Grove 1990.

[10] Petrov, V.V., Sums of Independent Random Variables, Springer-Verlag, Berlin 1975.

[11] Rachev, S.T., Probability Metrics and the Stability of Stochastic Models, J. Wiley \& Sons, Chichester 1991.

[12] Senatov, V.V., Uniform estimates of the rate of convergence in the multi-dimensional central limit theorem, Theory Prob. Appl. 25 (1980), 745-759.

[13] Zolotarev, V., Ideal metrics in the problems of probability theory, Austral. J. Statist. 21 (1979), 193-208. 


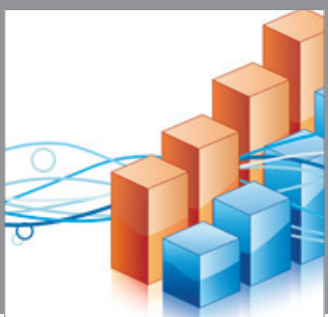

Advances in

Operations Research

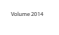

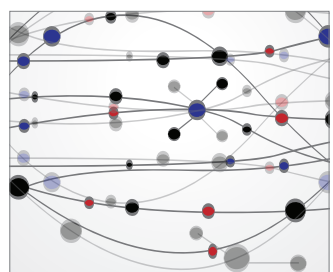

\section{The Scientific} World Journal
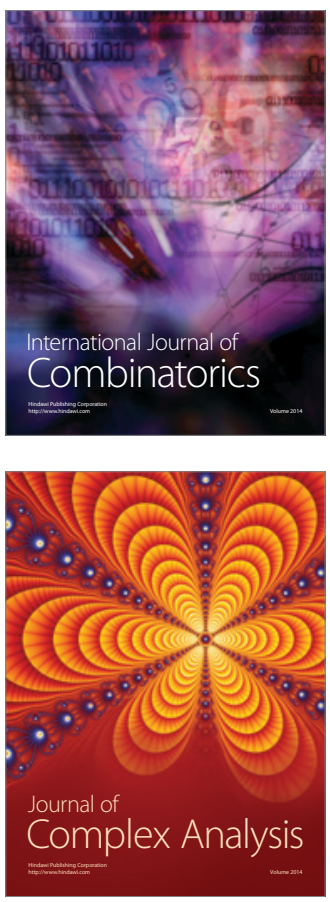

International Journal of

Mathematics and

Mathematical

Sciences
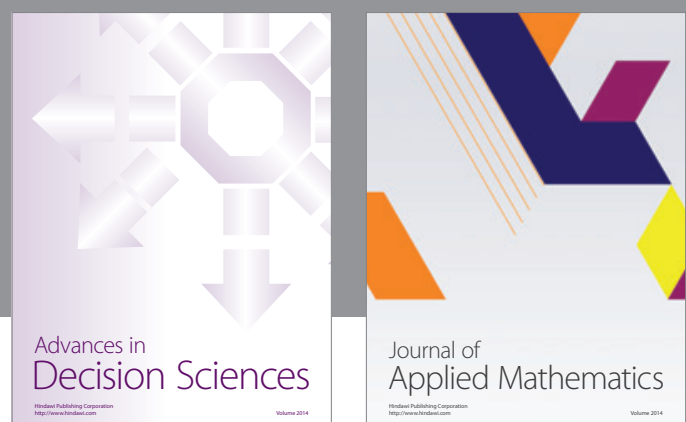

Journal of

Applied Mathematics
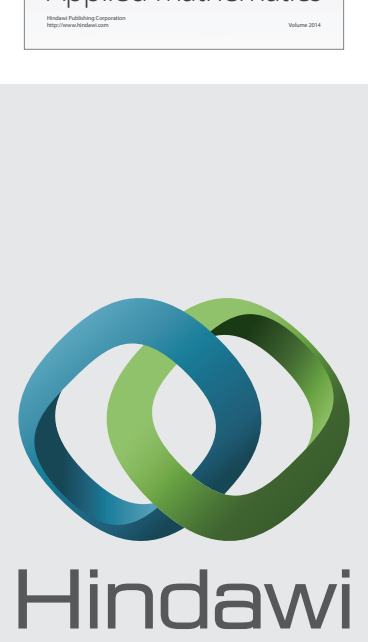

Submit your manuscripts at http://www.hindawi.com
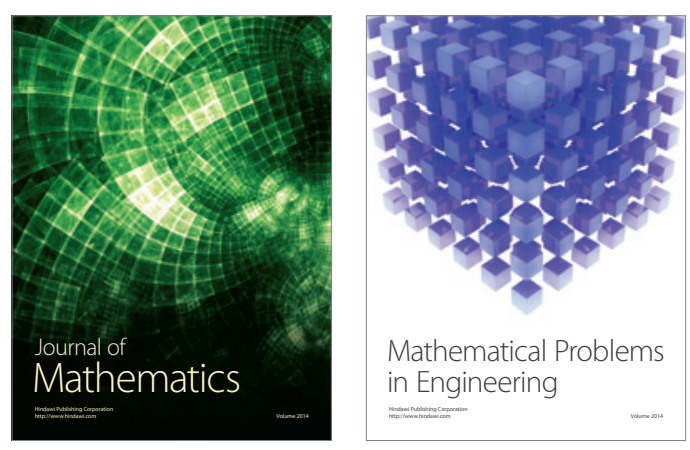

Mathematical Problems in Engineering
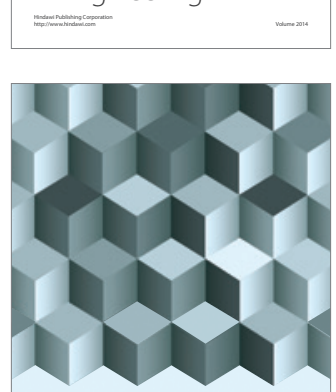

Journal of

Function Spaces
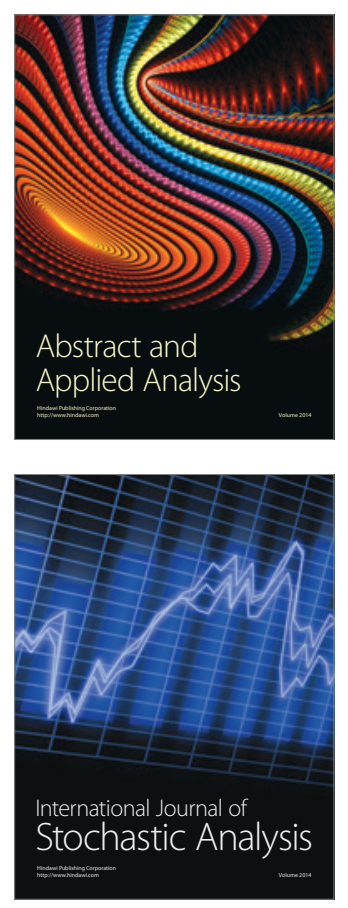

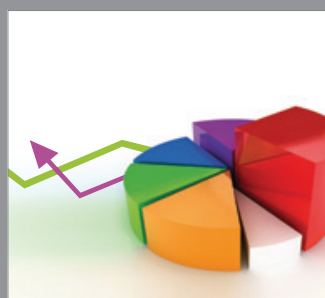

ournal of

Probability and Statistics

Promensencen
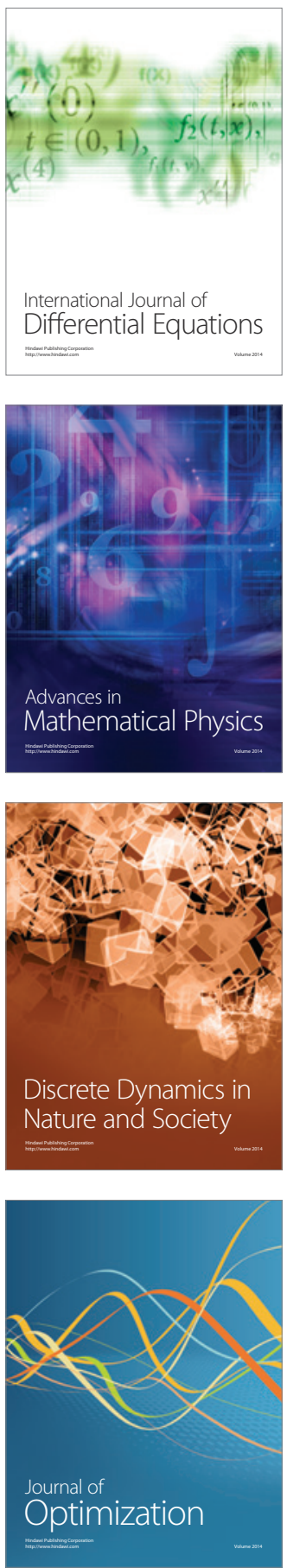